\title{
High diagnostic performance of a novel reverse transcription loop-mediated isothermal amplification (RT-LAMP) kit for the rapid detection of SARS-CoV-2
}

Alexander Domnich ( $\nabla$ alexander.domnich@hsanmartino.it )

Ospedale Policlinico San Martino

Andrea Orsi

University of Genoa

Donatella Panatto

University of Genoa

Vanessa De Pace

Ospedale Policlinico San Martino

Valentina Ricucci

Ospedale Policlinico San Martino

Patrizia Caligiuri

Ospedale Policlinico San Martino

Giulia Guarona

Ospedale Policlinico San Martino

Valerio Chessa

Ospedale Policlinico San Martino

Diego Ferone

University of Genoa

Simona Boccotti

University of Genoa

Bianca Bruzzone

Ospedale Policlinico San Martino

Giancarlo Icardi

University of Genoa

\section{Research Article}

Keywords: COVID-19, SARS-CoV-2, RT-LAMP, RT-PCR, Diagnostic accuracy

Posted Date: October 6th, 2021 
DOI: https://doi.org/10.21203/rs.3.rs-956125/v1

License: (c) (1) This work is licensed under a Creative Commons Attribution 4.0 International License. Read Full License 


\section{Abstract}

Although the reverse transcription polymerase chain reaction (RT-PCR) is considered a standard-of-care assay for the laboratory diagnosis of SARS-CoV-2, several limitations of this method have been described. Reverse transcription loop-mediated isothermal amplification (RT-LAMP) is an alternative molecular assay and is potentially able to overcome some intrinsic shortcomings of RT-PCR. In this study, we evaluated the diagnostic performance of the novel HG COVID-19 RT-LAMP assay. In this retrospective analysis, a total of 400 routinely collected leftover nasopharyngeal samples with a known RT-PCR result were tested by means of the HG COVID-19 RT-LAMP assay. The overall sensitivity and specificity values of HG COVID-19 RT-LAMP versus RT-PCR were $97.0 \%$ (95\% Cl: 93.6-98.9\%) and 98.5\% (95\% Cl: 95.7$99.7 \%)$, respectively. Inter-assay agreement was almost perfect $(K=0.96)$. Concordance was perfect in samples with high viral loads (cycle threshold $<30$ ). The average time to a positive result on RT-LAMP was $17 \mathrm{~min}$. HG COVID-19 RT-LAMP is a reliable molecular diagnostic kit for detecting SARS-CoV-2, and its performance is comparable to that of RT-PCR. Shorter turnaround times and the possibility of performing molecular diagnostics in the point-of-care setting make it a valuable option for facilities without sophisticated laboratory equipment.

\section{Introduction}

The precise laboratory diagnosis of SARS-CoV-2 is crucial to tackling the ongoing COVID-19 pandemic. The laboratory diagnosis of both symptomatic and asymptomatic SARS-CoV-2 cases can be achieved by means of both direct (detection of the viral RNA or antigens) and indirect (detection of specific antibodies) approaches [1-3].

The reverse transcription polymerase chain reaction (RT-PCR) is considered the standard method for the laboratory diagnosis of SARS-CoV-2 infection, and several protocols for its execution have been developed $[1,4,5]$. However, while the specificity of RT-PCR is deemed high [6], some issues regarding its sensitivity have been reported. Indeed, a systematic review by Arevalo-Rodriguez et al. [7] has shown that the false negativity rate (defined as initial negative result followed by a positive result) may vary from $1.8-58 \%$ (median of $11 \%$ ). Other potential shortcomings of the RT-PCR assay include the need for both qualified personnel and sophisticated laboratory equipment, its still suboptimal turnaround time and its relatively high per-sample costs [3].

In order to overcome the above-mentioned intrinsic limits of RT-PCR, some alternative direct methods have been promptly developed [1-3]. Among these, antigen-detecting rapid diagnostic tests (Ag-RDTs) have become popular, and dozens of kits have been commercialized. However, as shown by a recent Cochrane review [8], the diagnostic performance of Ag-RDTs is highly variable and depends on several factors, including, for example, viral load, presence or absence of COVID-19 symptoms, time since the onset of symptoms, and brand. 
The loop-mediated isothermal amplification (LAMP) assay is another alternative to RT-PCR for the molecular diagnosis of SARS-CoV-2. LAMP of DNA was originally described in 2000 [9] and since that time this method has gained prominence as a rapid, accurate and cost-effective diagnostic method for a variety of pathogens $[10,11]$. Typical LAMP reagents comprise salts, nucleotides, DNA polymerase and a set of 4-6 primers, including loop primers, forward and reverse inner primers and outer primers [12]. Reverse transcription LAMP (RT-LAMP) protocols have been developed to detect RNA virus sequences by adding a heat-stable reverse transcriptase enzyme to the LAMP mixture [13]. Compared with RT-PCR, the LAMP technique can rapidly amplify and produce up to 100 times more nucleic acid copies in isothermal conditions of $60-65^{\circ} \mathrm{C}[12]$.

The first available data suggest that RT-LAMP assays have a high diagnostic performance in detecting SARS-CoV-2. In particular, a recent systematic review and meta-analysis [14] showed pooled sensitivity values of RT-LAMP versus RT-PCR of $94 \%$ (95\% Cl: $90-96 \%$ ) and 78\% (95\% Cl: 65-87\%) for extracted and fresh samples, respectively. Specificity was 100\% (95\% Cl: $99-100 \%)$ and $96 \%$ (95\% Cl: $95-99 \%)$ for extracted and fresh specimens, respectively.

The HG COVID-19 assay (HiberGene Diagnostics, Republic of Ireland) is among the first commercially available CE-certified RT-LAMP assays intended for the rapid molecular diagnosis of SARS-CoV-2. The development and commercialization of this assay were supported by the Horizon 2020 Research and Innovation program [15]. The objective of the present study was to assess the relative (compared with RTPCR) diagnostic performance of the HG COVID-19 assay in routinely processed nasopharyngeal (NP) specimens.

\section{Materials And Methods}

\section{Reporting quality}

For the purpose of reporting, we adopted the STARD (Standards for Reporting of Diagnostic Accuracy Studies) statement [16]. The checklist is available in Supplementary File 1.

\section{Overall study design, sampling and setting}

The NP samples analyzed in this study came from routine SARS-CoV-2 molecular diagnostics and were collected between 8th April and 9th May 2021. All samples were processed at the regional reference laboratory for COVID-19 diagnostics, located at San Martino Policlinico Hospital, Hygiene Unit (Genoa, Italy). This laboratory performs SARS-CoV-2 RT-PCR on both in- and outpatient specimens, independently of the purpose of testing (laboratory confirmation of symptomatic cases, screening, clinical follow-up, etc.). No clinical data associated with a given sample were available. All samples were taken by means of a flocked swab kit and were eluted in universal transport medium (UTM, Copan Diagnostics Inc, US).

The index test was the HG COVID-19 assay, while the reference test was RT-PCR, which is currently considered the "gold standard" technique for the laboratory diagnosis of SARS-CoV-2 [1, 4, 5]. Following a 
successful RT-PCR run, the anonymized leftover samples were tested by means of RT-LAMP and therefore the positivity status was known beforehand. All RT-PCR tests were executed within 8 hours of the arrival of specimens at the laboratory.

Any NP samples with a known RT-PCR readout were potentially eligible for the study. Specimens showing $C t$ values $\leq 40$ were deemed positive. This cut-off was chosen on the basis of the RT-PCR assay available at our laboratory and considers samples with $\mathrm{Ct} \leq 40$ to be positive [17].

The sample size of 400 (200 positive and 200 negative RT-PCR samples) specimens was judged sufficiently powered to assess the diagnostic performance of the HG COVID-19 assay. Indeed, according to the Foundation for Innovative New Diagnostics [18], a minimum of 100 RT-PCR positive and 100 RTPCR negative samples is needed for the comparative evaluation of Ag-RDTs. Two-hundred routinely analyzed positive specimens were collected by means of quota sampling. Specifically, on the basis of RTPCR Ct values, positive samples were first categorized into four groups: (i) <20; (ii) 20-24.9; (iii) 25-29.9 and (iv) $\geq 30$, and consecutively fulfilled in a 1:1:1:1 ratio (i.e., 50 specimens per group). Given that the HD COVID RT-LAMP assay targets the nucleoprotein $(\mathrm{N})$ gene, samples were categorized according to the RTPCR Ct value of this gene. All 200 negative samples were gathered according to convenience.

During the study period, most ( $>90 \%$ ) detections (as shown by a random sample of specimens sequenced by our laboratory on regular basis) were attributable to the Alpha variant of concern of SARS-CoV-2. For the propose of this study, we further analyzed a sample of 64 RT-PCR-positive specimens $(\mathrm{Ct}<30)$ to determine their lineage. This was done by means of the RT-PCR-based Variant Catcher system by Clonit (Italy), which is able to distinguish between the so-called wild-type (Wuhan-like) and Alpha and Beta/Gamma variants of concern.

\section{Real-time reverse transcription polymerase chain reaction (RT-PCR)}

As per the internal SARS-CoV-2 diagnostic protocol adopted by the San Martino Policlinico Hospital, all NP samples were processed by means of a validated [19] unheated RNA extraction-free RT-PCR method. We have previously shown [19] that this technique displays perfect agreement with a traditional extraction-based approach. Briefly, an input volume of $5 \mu$ of specimen was first diluted (1:3) and set up for RT-PCR. RT-PCR was run on a CFX96 instrument (Bio-Rad Laboratories, US) by using the Allplex 2019$\mathrm{nCoV}$ (Seegene Inc., South Korea) assay. This multiplex assay is able to simultaneously detect three gene targets, namely N, RNA-dependent RNA-polymerase (RdRp)/spike (S) and envelope (E) regions.

Amplification was performed at $50^{\circ} \mathrm{C}$ for $20 \mathrm{~min}, 95^{\circ} \mathrm{C}$ for $15 \mathrm{~min}, 45$ cycles at $95^{\circ} \mathrm{C}$ for $10 \mathrm{~s}, 60^{\circ} \mathrm{C}$ for $15 \mathrm{~s}$ with first acquisition and $72^{\circ} \mathrm{C}$ for $10 \mathrm{~s}$ with second acquisition on the CFX 96 thermal cycler. A total of 5 $\mu$ of the extracted RNA in a final volume of $20 \mu$ was used for each reaction.

For the purpose of this study, only "valid" (i.e., when the internal control successfully amplified) RT-PCR samples were eligible.

\section{HG COVID-19 assay}


The index test was the HG COVID (HiberGene Diagnostics, Republic of Ireland) assay. This is a LAMPbased assay that targets the highly conserved $\mathrm{N}$ region. The reaction strips contain both complete lyophilized reaction mixes for SARS-CoV-2 and an extraction control, which is used to demonstrate the absence of inhibitors. The HG COVID assay can be performed in two formats: (i) direct (i.e., from the fresh sample) and (ii) extracted. In this validation study, the extracted option was used. Total RNA of NP specimens was extracted by means of the STARMag Universal Cartridge Kit (Seegene Inc., South Korea) on the automated Nimbus IVD (Seegene Inc., South Korea) platform. The extracts obtained were eluted in $140 \mu$ of the viral elution buffer provided with the kit.

Subsequently, a total of $25 \mu \mathrm{l}$ of the lysed samples obtained was added to target and control wells, vortexed for $5 \mathrm{~s}$ and centrifuged for $10 \mathrm{~s}$ at $3000 \mathrm{rcf}$. The reaction was run on the HG Swift instrument (HiberGene Diagnostics, Republic of Ireland).

The final readout is qualitative and may be displayed as "positive", "negative" or "inconclusive". According to the manufacturer, positive results are available in $<30 \mathrm{~min}$, while negative results can be recorded in $<60 \mathrm{~min}$. For positive samples, the time to result was automatically collected. In the case of an inconclusive result, the test was repeated.

\section{Data analysis}

Categorical variables are expressed as proportions with $95 \% \mathrm{Cls}$, and approximately normally distributed continuous variables as means with standard deviations (SDs). Chi-square and $t$ tests were used to compare proportions and continuous variables, respectively. There were no missing data (Supplementary File 2).

The diagnostic performance of the HG COVID assay was compared with the output of RT-PCR in terms of overall diagnostic accuracy, Cohen's $k$, sensitivity and specificity. These statistics were also calculated according to the RT-PCR Ct values for the $\mathrm{N}$ gene.

For true positive samples, the average (SD) time to the result of the HG COVID assay was calculated. Pearson's r coefficient was used to establish a correlation between the RT-PCR Ct value for the $\mathrm{N}$ gene and time to the result provided by RT-LAMP.

The raw dataset used is available in Supplementary File 2. All analyses were performed in R stats packages v. 4.0.3 [20].

\section{Results}

A total of 400 (200 positive and 200 negative) RT-PCR NP swabs were analyzed in the present study (Supplementary File 3). The mean age of subjects was 50.4 (SD 21.8) years; $52.5 \%$ (95\% Cl: $47.5-57.5 \%$ ) were females. RT-PCR-positive and -negative subjects were similar in terms of age [49.7 (SD 21.8) vs 51.0 (SD 21.8) years, respectively; $P=0.56]$ and sex [50.0\% (95\% Cl: $42.9-57.1 \%)$ vs $55.0 \%$ (95\% Cl: $47.8-$ $62.0 \%)$ of females, respectively; $P=0.37$. Among RT-PCR-positive samples, Ct values for the $\mathrm{N}$ gene 
ranged from 15 to 39 , with an average of 24.9 (SD 5.7). Among the samples tested for lineages $(N=64)$, all but one [98.4\% (95\% Cl: $91.6-100 \%)]$ belonged to the Alpha variant of concern.

Table 1 reports the raw data on the comparison between the RT-PCR and HG COVID RT-LAMP assays in terms of SARS-CoV-2 detection in NP samples. Briefly, a total of nine [2.3\% (95\% Cl: $1.0-4.2 \%)]$ discordant results were documented: six [3.0\% (95\% Cl: $1.1-6.4 \%)]$ were judged false negatives, while the other three [1.5\% (95\% Cl: $0.3-4.3 \%)]$ were false positives. Therefore, the overall diagnostic accuracy, sensitivity and specificity values were $97.8 \%, 97.0 \%$ and $98.5 \%$, respectively. Moreover, the overall interassay concordance was judged almost perfect $(\kappa=0.96)$ (Table 2$)$.

Table 1

Two-per-two table on the performance of the HG COVID RT-LAMP assay, as compared with RT-PCR*

\begin{tabular}{|llll|}
\hline RT-LAMP & \multicolumn{2}{l}{ RT-PCR } & \multicolumn{2}{c|}{ Total } \\
\cline { 2 - 3 } & Positive & Negative & \\
\hline Positive & $97.0(194)$ & $1.5(3)$ & $49.3(197)$ \\
\hline Negative & $3.0(6)$ & $98.5(197)$ & $50.7(203)$ \\
\hline Total & $50.0(200)$ & $50.0(200)$ & $100(400)$ \\
\hline * Results are reported as \% $(M)$ & \\
\hline
\end{tabular}

Table 2

Diagnostic performance indicators of the HG COVID RT-LAMP assay, as compared with RT-PCR

\begin{tabular}{|lll|}
\hline Parameter & Estimate & $\mathbf{9 5 \%} \mathbf{C l}$ \\
\hline Diagnostic accuracy, \% & 97.8 & $95.8-99.0$ \\
\hline Sensitivity, \% & 97.0 & $93.6-98.9$ \\
\hline Specificity, \% & 98.5 & $95.7-99.7$ \\
\hline Cohen's $K$ & 0.96 & $0.86-1$ \\
\hline
\end{tabular}

The nine discordant results were then analyzed in detail. All six NP swabs that tested negative on RT-PCR had $\mathrm{N}$ gene $\mathrm{Ct}$ values $>30$ (range: $31-38$ ). Indeed, as shown in Table 3 , the relative sensitivity of the HG COVID assay was $100 \%$ for NP samples with $\mathrm{Ct}<30$ and $97.0 \%$ for those $\leq 39$. By contrast, of the three false positive NP samples, two had tested positive on RT-PCR 1-3 weeks earlier. 
Table 3

Sensitivity of the HG COVID RT-LAMP assay, as compared with RT-PCR, by N gene cycle threshold value

\begin{tabular}{|lll|}
\hline $\mathbf{N}$ gene cycle threshold $(\boldsymbol{M})$ & Estimate, \% & $\mathbf{9 5 \%} \mathbf{~ C l}$ \\
\hline$<20(50)$ & 100 & $92.3-100$ \\
\hline$<25(100)$ & 100 & $96.4-100$ \\
\hline$<30(150)$ & 100 & $97.6-100$ \\
\hline$<33(178)$ & 99.4 & $96.9-100$ \\
\hline$<36(195)$ & 97.4 & $94.1-99.2$ \\
\hline$\leq 39(200)$ & 97.0 & $93.6-98.9$ \\
\hline
\end{tabular}

Among the true positive samples $(N=194)$, the average time to result on the HG COVID RT-LAMP assay was 17.0 (SD 3.4) min. As expected, there was a clear $(r=0.82, P<0.001)$ relationship between the $\mathrm{N}$ gene $\mathrm{Ct}$ value and the time to the result of the HG COVID assay (Fig. 1). Indeed, the average time to result for samples with high viral loads (Ct <20) was only 13.9 (SD 1.9) min.

\section{Discussion}

This study is among the first independent on-field evaluations of the HG COVID-19 RT-LAMP assay. Our findings confirmed the high diagnostic performance of this assay in detecting SARS-CoV-2 in NP samples with a wide (15-39) range of Ct values. We also demonstrated that the recent predominance of the Alpha variant of concern did not affect the analytical performance of the kit. Indeed, the HG COVID-19 assay targets the highly conserved $\mathrm{N}$ region, while the novel variants of concern present key mutations in the $\mathrm{S}$ region. These mutations may affect the diagnostic performance of RT-PCR; indeed, so-called "S-gene target failure" is very common in samples positive for the Alpha variant of concern [21, 22]. Finally, our study is the first to validate the HG COVID-19 assay on an alternative extraction platform, which shows that RT-LAMP can be run successfully on different types of laboratory equipment.

According to the manufacturer's instructions for use, the relative (vs RT-PCR) sensitivity and specificity values of the HG COVID extracted format in symptomatic patients are $100 \%$ (95\% Cl: $84.5-100 \%$ ) and $100 \%$ (95\% Cl: $89.1-100 \%)$, respectively. In asymptomatic individuals, these parameters are $88.5 \%$ (95\% Cl: $75.9-95.2 \%)$ and $97.1 \%$ (95\% Cl: 92.9-98.9\%), respectively. Considering that our sample included both symptomatic and asymptomatic subjects, our findings are consistent with the on-label analytical performance and testify to the generalizability of our results.

Our results are also in line with the meta-analytical estimates obtained by Subsoontorn et al. [14]. In their systematic review, the pooled ( $N=26$ studies) sensitivity and specificity values of the extracted RT-LAMP format versus RT-PCR were $94 \%$ (95\% Cl: $90-96 \%)$ and 100\% (95\% Cl: $99-100 \%)$, respectively. When the analysis was restricted to high viral load samples $(\mathrm{Ct}<30)$, the pooled estimates $(N=10$ studies) were 
100\% (95\% Cl: 89-100\%) and 100\% (95\% Cl: 99-100\%), respectively [14]. In our study, while some false negatives with high RT-PCR Ct values (>30) were expected, some comments on the "false positives" should be made. It has been suggested that some non-specific amplifications may occur during RT-LAMP [23]. We documented a total of three false positive results; of these, however, two subjects had proved positive on RT-PCR 1-3 weeks earlier. On the one hand, it is well-known that the RT-LAMP technique can amplify up to 100 times more RNA copies than RT-PCR [12]. On the other hand, the RT-PCR assay used in this study (Allplex 2019-nCoV; Seegene Inc., South Korea) considers samples with $\mathrm{Ct} \leq 40$ to be positive [17]. It is therefore likely that the two above-mentioned subjects with false positive results could have had Ct values over 40. In turn, this may also mean that the reported specificity of the HG COVID-19 assay might have been underestimated. There is an ongoing debate on the association between infectiousness and RT-PCR Ct values. It is generally believed that infectiousness is significantly lower for clinical specimens with $\mathrm{Ct}$ values $>30[24,25]$. However, a substantial number of samples with $\mathrm{Ct}>35$ may still produce a viable virus [26-28]. Moreover, Ct values across different RT-PCR protocols may vary significantly [19].

The possibility of rapidly obtaining a precise result makes RT-LAMP technology an attractive point-of-care or near-the-patient tool. This feature of RT-LAMP resembles that of Ag-RDTs. The World Health Organization [29] recommends that SARS-CoV-2 Ag-RDTs should have sensitivity and specificity values of at least $80 \%$ and $97 \%$, respectively, while the European Centre for Disease Control and Prevention [24] has suggested that the sensitivity of point-of-care tests should be at least $90 \%$. The HG COVID assay satisfies these criteria. An assessment of several Ag-RDTs recently performed at our laboratory [30] showed overall sensitivity of $78.7 \%$ (95\% Cl: $73.2-83.3 \%$ ); this, however, varied significantly by Ct value and brand. We concluded that Ag-RDTs are convenient for screening purposes in moderate-to-high intensity settings [30]. In the present study, the analytical performance of the HG COVID-19 assay was comparable to that of RT-PCR; the assay may therefore be an alternative molecular diagnostic tool for SARS-CoV-2 detection, independently of the purpose and the viral epidemiology. On the other hand, it is likely that not all commercially available RT-LAMP kits perform well. For instance, a real-world evaluation of the diagnostic performance of the Isopollo COVID-19 RT-LAMP assay (M Monitor, South Korea) showed a sensitivity of only 61.9\% [31]. Independent and setting-specific pilot evaluations would therefore be beneficial before the widespread implementation of RT-LAMP assays.

Like that of most available Ag-RDTs, the HG COVID readout is qualitative. However, the time to threshold is a good proxy of viral load. Indeed, we observed a strong linear association between the HG COVID-19 time to result and RT-PCR Ct values. Results that are available in less than 15-20 min are highly suggestive of high viral loads $(\mathrm{Ct}<30)$.

Our study is not without limitations. First, for ethical reasons, we were not able to link the RT-PCR readout to the clinical characteristics of patients (e.g., presence of symptoms, days after the onset of symptoms, etc). According to the manufacturer's instructions for use, the performance of the HG-COVID assay is better in symptomatic cases, especially when the test is performed soon after the onset of symptoms. Second, we cannot completely rule out misclassification bias. Although RT-PCR is currently considered 
the "gold standard" assay for the laboratory diagnosis of both symptomatic and asymptomatic cases [1, 4, 5], its sensitivity is not perfect [7]. In our study, two samples from patients with previously confirmed SARS-CoV-2 infection were positive on RT-LAMP but negative on RT-PCR. This means that, in some instances, RT-LAMP may be more sensitive than RT-PCR, and that the reported relative specificity of RTLAMP might have been underestimated. Finally, the study was carried out in a period when Delta variant of concern did not circulate in Italy. We, however believe that this possible limitation has a limited impact on the study conclusions for two reasons. First, the HG COVID assay targets the highly conserved N region, while the key mutations of the Delta variant are located in the $S$ region. Second, our subsequent routine use of the HG COVID assay was able to detect isolates belonging to the Delta variant (results not shown).

In conclusion, HG COVID-19 RT-LAMP is a reliable assay for the molecular diagnosis of SARS-CoV-2 in NP samples, and yields a final diagnosis in less time than RT-PCR. RT-LAMP technology is promising for use in small and medium-sized hospitals, emergency departments and general practices without sophisticated laboratory equipment.

\section{Declarations}

Acknowledgements The authors thank Bernard Patrick for his linguistic review of the manuscript.

Funding This project received funding from the European Union's Horizon 2020 research and innovation programme under grant agreement No 101003713. This study reflects only the author's view and the European Commission is not responsible for any use that may be made of the information it contains.

Availability of data and materials The raw data are in available in Supplementary Materials.

Conflict of interest The authors declare no conflict of interest regarding this publication.

Ethics statement The study was conducted according to the guidelines of the Declaration of Helsinki. Ethical review and approval were not required, as this retrospective analysis was based on routine RT-PCR COVID-19 testing. RT-LAMP testing was performed on anonymized leftover nasopharyngeal samples.

\section{References}


1. Venter, M. \& Richter, K. Towards effective diagnostic assays for COVID-19: a review. J Clin Pathol, 73, 370-377 https://doi.org/10.1136/jclinpath-2020-206685 (2020).

2. Ravi, N., Cortade, D. L., Ng, E. \& Wang, S. X. Diagnostics for SARS-CoV-2 detection: A comprehensive review of the FDA-EUA COVID-19 testing landscape. Biosens Bioelectron, 165, 112454 (2020).

3. Russo, A. et al. Current status of laboratory diagnosis for COVID-19: A narrative review. Infect Drug Resist, 13, 2657-2665 https://doi.org/10.1016/j.bios.2020.112454 (2020).

4. Böger, B. et al. Systematic review with meta-analysis of the accuracy of diagnostic tests for COVID19. Am J Infect Control, 49, 21-29 https://doi.org/10.1016/j.ajic.2020.07.011 (2021).

5. Caruana, G. et al. Diagnostic strategies for SARS-CoV-2 infection and interpretation of microbiological results. Clin Microbiol Infect, 26, 1178-1182 https://doi.org/10.1016/j.cmi.2020.06.019 (2020).

6. Watson, J., Whiting, P. F. \& Brush, J. E. Interpreting a covid-19 test result. BMJ, 369, m1808 https://doi.org/10.1136/bmj.m1808 (2020).

7. Arevalo-Rodriguez, I. et al. False-negative results of initial RT-PCR assays for COVID-19: A systematic review. PLoS One, 15, e0242958 https://doi.org/10.1371/journal.pone.0242958 (2020).

8. Dinnes, J. et al. Rapid, point-of-care antigen and molecular-based tests for diagnosis of SARS-CoV-2 infection. Cochrane Database Syst Rev, 3, CD013705 https://doi.org/10.1002/14651858.cd013705.pub2 (2021).

9. Notomi, T. et al. Loop-mediated isothermal amplification of DNA. Nucleic Acids Res, 28, E63 https://doi.org/10.1093/nar/28.12.e63 (2000).

10. Kashir, J. \& Yaqinuddin, A. (2020) Loop mediated isothermal amplification (LAMP) assays as a rapid diagnostic for COVID-19. Med Hypotheses 2020;141:109786. https://doi.org/10.1016/j.mehy.2020.109786

11. Mori, Y. \& Notomi, T. Loop-mediated isothermal amplification (LAMP): a rapid, accurate, and costeffective diagnostic method for infectious diseases. J Infect Chemother, 15, 62-69 https://doi.org/10.1007/s10156-009-0669-9 (2009).

12. Thompson, D. \& Lei, Y. Mini review: Recent progress in RT-LAMP enabled COVID-19 detection. Sensors and Actuators Reports, 2, 100017 https://doi.org/10.1016/j.snr.2020.100017 (2020).

13. Augustine, R. et al. Loop-mediated isothermal amplification (LAMP): A rapid, sensitive, specific, and cost-effective point-of-care test for coronaviruses in the context of COVID-19 pandemic. Biology (Basel), 9, 182 https://doi.org/10.3390/biology9080182 (2020).

14. Subsoontorn, P., Lohitnavy, M. \& Kongkaew, C. The diagnostic accuracy of isothermal nucleic acid point-of-care tests for human coronaviruses: A systematic review and meta-analysis. Sci Rep, 10, 22349 https://doi.org/10.1038/s41598-020-79237-7 (2020).

15. Hibergene \& HG COVID-19. (2020). https://hibergene.com/products/respiratory/hg-covid-19/. Accessed 27 Sept 2021 
16. Cohen, J. F. et al. STARD 2015 guidelines for reporting diagnostic accuracy studies: explanation and elaboration. BMJ Open, 6, e012799 https://doi.org/10.1136/bmjopen-2016-012799 (2016).

17. Seegene Allplex 2019-nCoV assay (version 2.2; April 15th, 2021) (Cat no. RP10250X/RP10252W). Instructions for use (2021) https://www.fda.gov/media/137178/download. Accessed 27 Sept 2021

18. Foundation for Innovative New Diagnostics (FIND). Comparative evaluation of lateral flow assay tests that directly detect antigens of SARS-CoV-2 (2020). https://www.finddx.org/wpcontent/uploads/2020/04/20200421-COVID-Ag-RDT-Evaluation-Synopsis.pdf Accessed 27 Sept 2021

19. Domnich, A. et al. Evaluation of extraction-free RT-qPCR methods for SARS-CoV-2 diagnostics. Arch Virol, 166, 2825-2828 https://doi.org/10.1007/s00705-021-05165-0 (2021).

20. R Core Team. R: A language and environment for statistical computing (2020) Available from: http://www.R-project.org/. Accessed 27 Sept 2021

21. Brown, K. A. et al. S-gene target failure as a marker of variant B.1.1.7 among SARS-CoV-2 isolates in the greater Toronto area, December 2020 to March 2021. JAMA, 325, 2115-2116 https://doi.org/10.1001/jama.2021.5607 (2021).

22. Borges, V. et al. Tracking SARS-CoV-2 lineage B.1.1.7 dissemination: insights from nationwide spike gene target failure (SGTF) and spike gene late detection (SGTL) data, Portugal, week 492020 to week 3 2021. Euro Surveill, 26, 2100131 https://doi.org/10.2807/1560-7917.es.2021.26.10.2100130 (2021).

23. Moehling, T. J., Choi, G., Dugan, L. C., Salit, M. \& Meagher, R. J. LAMP diagnostics at the point-of-care: Emerging trends and perspectives for the developer community. Expert Rev Mol Diagn, 21, 43-61 (2021).

24. European Centre for Disease Prevention and Control (ECDC). Options for the use of rapid antigen tests for COVID-19 in the EU/EEA and the UK. 19 November 2020 (2020) https://www.ecdc.europa.eu/sites/default/files/documents/Options-use-of-rapid-antigen-tests-forCOVID-19.pdf Accessed 27 Sept 2021

25. Rao, S. N., Manissero, D., Steele, V. R. \& Pareja, J. A systematic review of the clinical utility of cycle threshold values in the context of COVID-19. Infect Dis Ther, 9, 573-586 https://doi.org/10.1007/s40121-020-00324-3 (2020).

26. Yamada, S. et al. Assessment of SARS-CoV-2 infectivity of upper respiratory specimens from COVID19 patients by virus isolation using VeroE6/TMPRSS2 cells. BMJ Open Respir Res, 8, e000830 https://doi.org/10.1136/bmjresp-2020-000830 (2021).

27. Singanayagam, A. et al. Duration of infectiousness and correlation with RT-PCR cycle threshold values in cases of COVID-19, England, January to May 2020. Euro Surveill, 25, 2001483 https://doi.org/10.2807/1560-7917.es.2020.25.32.2001483 (2020).

28. Romero-Gómez, M. P. et al. Ct value is not enough to discriminate patients harbouring infective virus. J Infect, 82, e35-e37 https://doi.org/10.1016/j.jinf.2020.11.025 (2021). 
29. World Health Organization (WHO). Antigen-detection in the diagnosis of SARS-CoV-2 infection using rapid immunoassays: Interim guidance (2020) https://www.who.int/publications/i/item/antigendetection-in-the-diagnosis-of-sars-cov-2infection-using-rapid-immunoassays Accessed 27 Sept 2021

30. Bruzzone, B. et al. Comparative diagnostic performance of rapid antigen detection tests for COVID-19 in a hospital setting. Int J Infect Dis, 107, 215-218 https://doi.org/10.1016/j.jij.2021.04.072 (2021).

31. Freire-Paspuel, B. \& Garcia-Bereguiain, M. A. Low clinical performance of the Isopollo COVID-19 detection kit (M Monitor, South Korea) for RT-LAMP SARS-CoV-2 diagnosis: A call for action against low quality products for developing countries. Int J Infect Dis, 104, 303-335 https://doi.org/10.1016/j.jijid.2020.12.088 (2021).

\section{Figures}

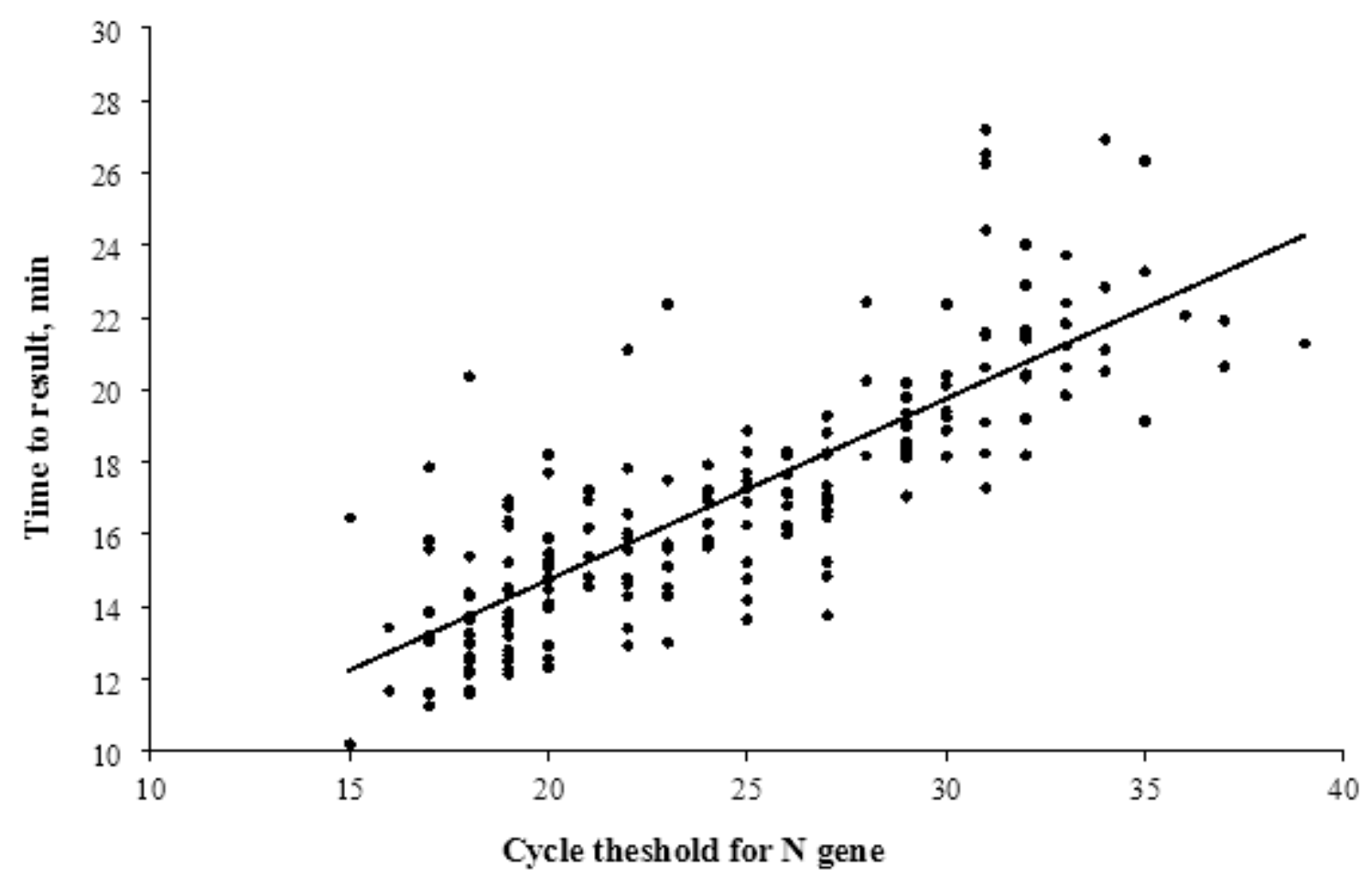

Figure 1

Correlation between $\mathrm{N}$ gene cycle threshold values and time to positive result on the HG COVID RT-LAMP assay

\section{Supplementary Files}

This is a list of supplementary files associated with this preprint. Click to download.

- SupplementaryFile1.pdf

- SupplementaryFile2.csv 
- SupplementaryFile3.pdf

Page 14/14 